\title{
Toward better understanding of the effect of fiber distribution on effective elastic properties of unidirectional composite yarns
}

\author{
M. Shah Mohammadi, M. Komeili, A.B. Phillion, A.S. Milani* \\ School of Engineering, University of British Columbia, Kelowna V1Y 1V7, Canada \\ *Corresponding author: Abbas S. Milani \\ E-mail: abbas.milani@ubc.ca
}

\begin{abstract}
A combined X-ray micro-computed tomography (XMT) and micro- Finite Element Analysis study is presented to quantify the effects of micro-scale random fiber distributions on the effective (homogenized) elastic properties of unidirectional (UD) yarns, as often used by designers for component-level computational modelling of composite structures. In addition, it is shown how the XMT artefacts can yield unreliable FE homogenization of the composite yarns by overestimating the stress transfer capacity between the material constituents. Finally, the microFEA modeling results under fiber distribution randomness are compared to the macro-level predictions such as the classical rule of mixture and Halpin-Tsai equations.
\end{abstract}

Keywords: Glass fibre yarns; Polymer-matrix composite; Effective elastic properties; Microfinite element analysis; Random fiber distribution; X-ray tomography

\section{Introduction}

Long-fiber reinforced polymers have gained significant attention in many industrial applications because of their low density, high specific stiffness and strength, and low thermal expansion [1]. Such composites are inherently multi-scale materials since their properties at the macro-scale are strongly affected by their fiber architecture and behaviour at lower scales. In general, a composite material has three distinguishable scales: (i) macro-scale which normally refers to a manufactured part, (ii) meso-scale which is an intermediate scale and refers to the yarn architecture, and (iii) micro-scale which concerns the assembly of a large number of fibers that make up an individual yarn [2]. 
Over the years, numerical simulation tools have been extensively utilized to investigate the behaviour of composites at different material scales [3]. One area of interest has been the prediction of macro-mechanical properties using micro-mechanics modelling, based on the known properties of the matrix and the reinforcement material phases [4]. This method is normally preformed through the analysis of a representative volume element (RVE) or a unit cell $[5,6,7]$. For different types of composites (e.g. unidirectional and textile composites), the mechanical properties of the material macrostructure can be simulated by periodically repeating such unit cells. Focusing on the case of unidirectional lamina, the repeating unit cell has been often defined in the literature assuming that the reinforcement has a uniform distribution and identical geometry [4]. However, not all the reinforcing phase constituents necessarily have the same geometry. In the case of glass fiber reinforced polymers (GFRPs), for example, depending on the supplier of the raw material, the fibers can have very different radii and the arrangement of fibers within the matrix is often non-periodic. Although these micro-level uncertainty effects will vary the properties of composite part at the macro-level [2], the available simulation tools do not often take such material inhomogeneity into account $[8,9]$, and designers in industry use average macro-material properties from given datasheets/handbooks. However, to obtain an accurate and reliable virtual model of a composite part at macro-level, the statistics of the microstructure must be characterized and incorporated using microscopy of actual specimens and a multi-scale analysis framework [10].

Due to advances in computer sciences and image processing technologies, image-based modelling, in which detailed models of specimens are created based on realistic geometry acquired via microscopy, has made significant advancements in the last decade. Three-dimensional (3D) image-based modelling is today possible using X-ray micro-computed tomographic microscopy (XMT) machines for image acquisition, followed by image processing and meshing using available software such as ScanIP ${ }^{\mathrm{TM}}$ (Simpleware, UK). The combination of XMT and image processing tools is a useful technique for modelling of complex hierarchical materials such as composites [11]. Blacklock et al. [10] have used statistical data obtained from XMT to generate virtual textile composite specimens rather than using the XMT image directly. More specifically, the textile reinforcement was presented as one-dimensional tow loci in 3D space, which was then incorporated into a binary model of textile composites. Simulation and XMT analysis of textile composite reinforcement deformation (e.g., biaxial tension and in-plane shear) at the mesoscopic scale was performed by Badel et al. [12] to characterize the 3D geometries of the deformed and 
un-deformed reinforcement, and particularly to provide a correct fiber distribution within a yarn model. In another study, Alghamdi et al. [11] presented a multi-scale 3D image-based model of a carbon/carbon $(\mathrm{C} / \mathrm{C})$ composite to evaluate its thermal diffusivity and Young's modulus. Specifically, two finite element (FE) models were created at different length scales; the microscale model was developed from scanning electron microscope (SEM) micrographs of the carbon tow, while the macro-scale model was developed from XMT images of the composite. The use of XMT images to create an image-based model of composites is advantageous because, due to its non-destructive nature, the XMT technique prevents the internal microstructure/features from being affected by sample preparation stages such as cutting of the specimen. However, a disadvantage in an image-based model of polymer-matrix composites is the similar absorption coefficients of the fibers and matrix phases, leading to similar grayscale levels and thus making fiber segmentation difficult [11]. The micron level spatial resolution of XMT images may also make it challenging to properly separating adjacent fibers, depending on the fiber diameter.

As outlined above, finite element (FE) modeling at the micro-level can be used to estimate the mechanical properties of a composite part at a macro-level; which is normally the level used by most designers in industry. On the other hand, the heterogeneity is an essential/inherent characteristic of composite materials [13-23]. Thus, 'homogenization' through numerical methods (e.g., FE) has been implemented by researchers as a capable method for predicting properties of the material in higher scales using results of analysis in lower scales. Homogenization starts by considering an RVE that is a subsystem of the entire composite part and can represent its typical structure fairly well $[13,14,15]$. For composite parts with an ideal periodic media, defining this unit cell is rather easy. However, the reality of fibers' random distribution inside the matrix is more challenging. In general, the selected RVE should include a reasonable number of micro-level features and represent overall fiber distribution. The effect of micro-level random features during homogenization of composites along with the effect of RVE size has been scarcely studied in the past.

In the present study, a combined XMT-FEA investigation has been carried out to characterize the effects of micro-scale fiber spatial distribution on the overall mechanical properties of a typical unidirectional (UD) fiberglass/polymer composite yarn. First, XMT has been used to image a consolidated UD yarn containing glass fibers. Then, a series of finite-element simulations were carried out on both randomized micro-scale RVEs (hereafter called "Randomized 
Unit Cell Analysis Library" or R-UCAL), as well as a full-size scanned macro-scale yarn specimen. Although many other uncertainly variables could be considered, the focus of this work is to characterize the effect of fiber radii as well as fiber centroid distribution on the ensuing effective properties of consolidated UD yarns at different volume fractions, when compared to theoretical (average) predictions. The assessment of the FE models generated by random reinforcement phases immersed in the matrix, versus those meshed based on the computed microtomography scans of the real material microstructure, has also been the topic of recent studies, although on different types of materials and properties (e.g., on a $\mathrm{Cr}-\mathrm{Al} 2 \mathrm{O} 3-\mathrm{Re}$ composite and its Young's modulus [24], or on a random chopped fiberglass composite and its Young's modulus as well as in-plane shear modulus [25], or on a short steel fibers composite and its equivalent orientation tensor [26]). Also recently a new approach for compressing and reconstructing complex information on the two-dimensional microstructures of disordered (random) particulate media has been introduced [27], by means of a finite set of tiles assembled via a stochastic tiling algorithm. The current case study has selected a long-fiber reinforced thermoplastic (LFRT) material along with the five elastic properties that are needed for its macro-level three-dimensional FE analysis as a transversely isotropic UD composite.

\section{Methodology}

\subsection{X-ray tomographic microscopy}

X-ray micro-computed tomography (XMT) was performed on a unidirectional (UD) yarn containing glass fibers embedded in polypropylene matrix, using a MicroXCT-400 (Zeiss ${ }^{\mathrm{TM}}$ ) instrument in order to visualize fiber distribution. During the XMT set-up, 2500 2-D radiographic projections were acquired at different angles through $360^{\circ}$ at an exposure time of $25 \mathrm{~s}$ for each radiograph. The projections were reconstructed to create a tomographic volume containing $1600 \times 1600 \times 500$ voxels at a voxel size of $0.53 \mu \mathrm{m}$. The volume image was then post-processed to separate the fiber and matrix phases and to extract geometrical information about the fiber distribution within the yarn using the advanced ImageJ and Avizo ${ }^{\circledR}$ packages. Due to imaging a UD yarn, all the cross-sectional slices were comparable. Thus, only a single slice, shown in Figure 1 , was used for further analysis. 


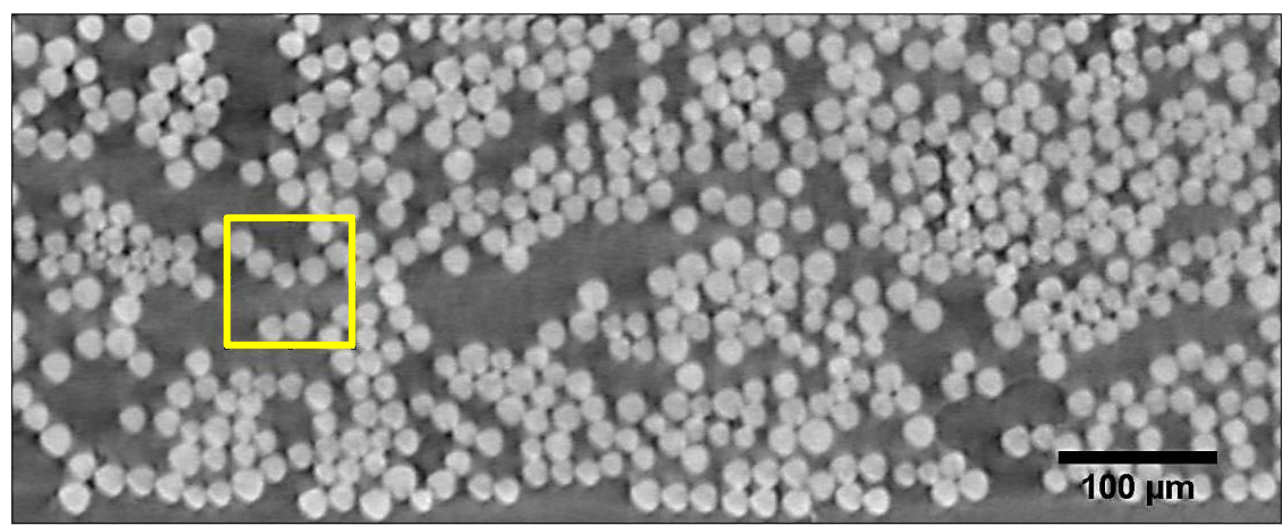

Figure 1 X-ray micro-computed tomography (XMT) image of a PP/glass UD yarn; the example of small unit cell/RVE extracted from the XMT image has been illustrated by a square box. In establishing the R-UCAL library, this box was randomly located at several locations to capture different local fiber distributions within the yarn.

\subsection{Finite Element Analysis of R-UCAL}

\subsubsection{Geometry and mesh}

A series of small representative volume elements (RVEs) with a fiber volume fraction ranging from 0.14 to 0.54 , shown in Figure 2, were randomly extracted from the XMT image of Figure 1 for the consolidated PP/glass UD composite yarn, in order to establish a Randomized Unit Cell Analysis Library (R-UCAL) with cell size of 150x150 pixels (0.08x0.08 mm). For each library element (i.e., individual unit cell as an idealized binary image similar to the study [28]), the coordinate and radius of individual fibers were extracted using a digitizer software (xyExtract) and an in-house developed Matlab ${ }^{\circledR}$ code, and then regenerated in Abaqus ${ }^{\circledR}$ as an FE mesh. 3D continuum elements were used to discretize the library elements. 

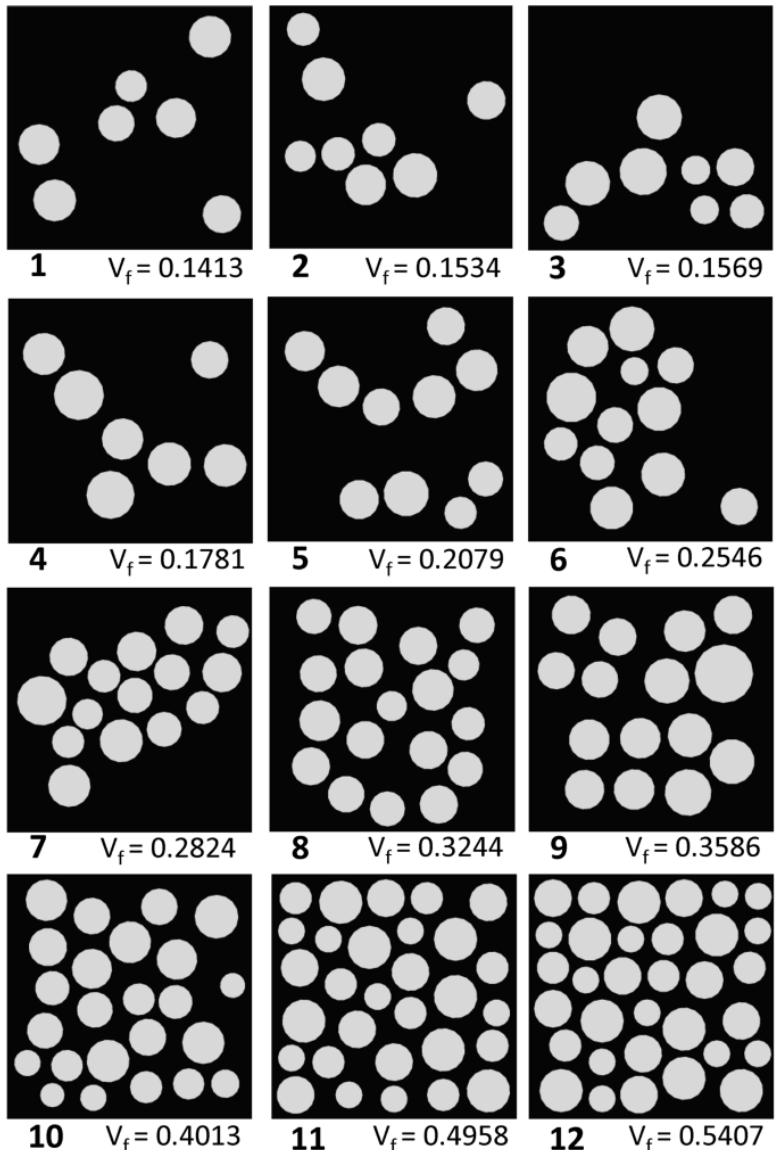

Figure 2 Small unit cells (R-UCAL library elements) extracted and regenerated from the actual scanned yarn, resulting in different volume fractions and fibre distributions. This library of unit cells was then used to obtain the effective mechanical properties and investigate the effect of fibre distribution on the yarn macro-level properties. The elements were also used to regenerate a random whole-yarn model in order to predict its effective mechanical properties and compare to the actual scanned yarn model properties.

\subsubsection{Simulation procedure}

In order to simulate deformation of the library elements, a series of finite element models was created and analyzed using the commercial FE package SIMULIA Abaqus as follows. The mechanical simulations were performed under the assumption of small deformation theory. It must be noted that the theoretical grounds laid down in subsequent subsections are suitable particularly when using commercial software. When building own in-house FE software, the formulation may be provided in a simpler way, e.g., without giving special attention to the Poisson's ration and shear stiffness effects [29], depending on the geometry or loading condition of structure. 


\section{(i) Periodic boundary conditions}

The boundary conditions that are applied to imitate the adjacent repetitive cells in RVE modeling are called periodic boundary conditions (PBCs) [15]. The basic principle of a periodic boundary condition relies on the fact that from a micro-level point of view, each point on one side of the RVE undergoes deformation identical to the point on the opposite side. In this way, an RVE can be used to generate the entire medium through repetition of a particular pattern and hence a single RVE can ideally represent the mechanical response of the entire media [16]. For instance, Figure 3 illustrates a general cube shaped RVE. If this RVE is in a periodic medium, surface $\Gamma_{j}^{+}$ on one side is attached to the $\Gamma_{j}^{-}$surface of another RVE right next to it. The displacement field condition can be rewritten as [15]:

$$
u_{i}(\boldsymbol{x})=u_{i, m}(\boldsymbol{x})+u_{i, p}(\boldsymbol{x})
$$

where $u_{i}(\boldsymbol{x})$ is the displacement vector and $u_{i, m}(\boldsymbol{x})$ and $u_{i, p}(\boldsymbol{x})$ are the macro-level/macroscopic and periodic/fluctuating component of the displacement vector, respectively. It can be argued that under a macroscopically uniform loading on the medium (where the average deformation remains the same over any arbitrary number of RVEs), $u_{i, p}(\boldsymbol{x})$ is the same for $\Gamma_{j}^{+}$and $\Gamma_{j}^{-}[16,17,18]$. Thus, $u_{p}\left(\Gamma_{j}^{+}\right)=u_{p}\left(\Gamma_{j}^{-}\right)$, which results in:

$$
\begin{gathered}
u_{i}\left(\Gamma_{j}^{+}\right)-u_{i}\left(\Gamma_{j}^{-}\right)=\left[u_{i, m}\left(\Gamma_{j}^{+}\right)+u_{i, p}\left(\Gamma_{j}^{+}\right)\right]-\left[u_{i, m}\left(\Gamma_{j}^{-}\right)+u_{i, p}\left(\Gamma_{j}^{-}\right)\right] \\
=u_{m}\left(\Gamma_{j}^{+}\right)-u_{m}\left(\Gamma_{j}^{-}\right),
\end{gathered}
$$

assuming that the macro-level/macroscopic strain tensor $\left(\bar{\varepsilon}_{i j}\right)$ for uniform loading is known. Additionally, for small deformations [16, 19]:

$$
u_{i}\left(\Gamma_{j}^{+}\right)-u_{i}\left(\Gamma_{j}^{-}\right)=\bar{\varepsilon}_{i j} \Delta x_{j}
$$

where $\Delta x_{j}$ is the vector that connects each point on $\Gamma_{j}^{+}$to its corresponding periodic point on $\Gamma_{j}^{-}$, and the repeated indices are for summation.

The condition imposed by Eq. (3) for periodic boundary conditions was applied to the deformation simulation of the R-UCAL elements through use of a Multi-Point Constraint (MPC) in Abaqus. To control the displacements on each of the periodic surfaces, three reference nodes 
$\left(R_{m}\right)$ were additionally created, outside of the volume enclosed by the library elements. The MPC formulation can be expressed as:

$$
u_{i}\left(\Gamma_{j}^{+}\right)-u_{i}\left(\Gamma_{j}^{-}\right)=\bar{\varepsilon}_{i j} \Delta x_{j}=u_{i}\left(R_{j}\right)
$$

The imposition of macro-level strains $\left(\bar{\varepsilon}_{i j}\right)$ on the RVE was carried out by adjusting the displacement degree of freedom, $i$, on the reference point, $j$, to match the applied deformation. The unconstrained displacement degrees of freedoms on the reference nodes were then evaluated after the analysis and transferred to their corresponding strain values. For example, uniaxial extension of $\bar{\varepsilon}_{11}$ along direction 1 was achieved by displacing $u_{1}$ in $R_{1}$ with a value of $\bar{\varepsilon}_{11} \Delta x_{1}$, where $\Delta x_{1}$ is the distance between $\Gamma_{1}^{+}$and $\Gamma_{1}^{-}$. Upon completion of the simulation, $u_{2}$ in $R_{2}$ was extracted to find $\bar{\varepsilon}_{22}=u_{2}\left(R_{2}\right) / \Delta x_{2}$. Similarly, the other loading modes were applied on the RVE, and the unconstrained degrees of freedom were used to find the resultant deformations. To ensure the symmetry condition in shear modes, $\bar{\varepsilon}_{i j}=\bar{\varepsilon}_{j i}$, two boundary conditions were imposed for each shear strain component. For example, in order to impose $\bar{\varepsilon}_{12}=\bar{\varepsilon}_{21}, u_{1}=\bar{\varepsilon}_{12} \Delta x_{2}$ was applied on $R_{2}$ while $u_{2}=\bar{\varepsilon}_{12} \Delta x_{1}$ was applied on $R_{1}$.
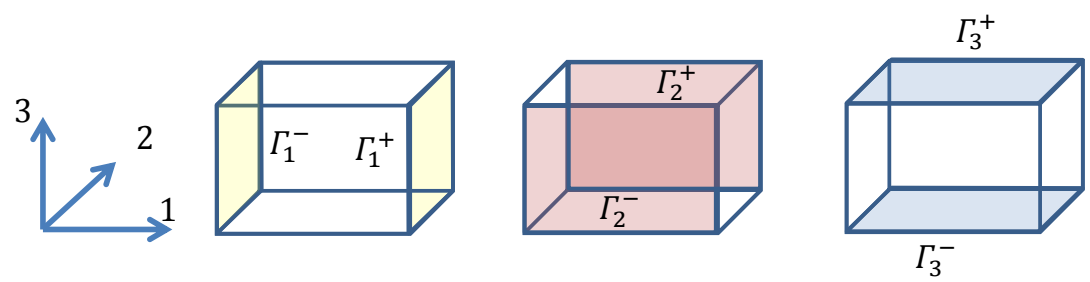

Figure 3 Periodic faces on a typical cubic RVE.

\section{(ii) Analysis procedure}

For each library element in the R-UCAL, the relation between the tensor of average strain and stress was determined in order to acquire the effective elastic mechanical properties of the composite yarn under random fiber distribution in the yarn's cross-section. The macro-level strains, $\bar{\varepsilon}_{i j}$, were either imposed as the loading condition or were read from the displacement degrees of freedom of the unconstrained reference nodes. Although an average level of stress, $\bar{\sigma}_{i j}$, can be found by averaging the stress level across all the elements in the RVE [16], a more convenient alternative would be a formulation based on the conservation of energy ( $W_{\text {ext }}=$ $\left.W_{\text {elastic }}\right)$. Under static condition and single mode loading, the conservation of energy for axial extension modes (i.e. applying $\varepsilon_{i i}$, no summation on $i$ ) is reduced to: 


$$
\begin{gathered}
W_{\text {ext }}=\int F_{k} d u_{k} \\
W_{\text {elastic }}=\frac{1}{2} \bar{E}_{i i} \bar{\varepsilon}_{i i}^{2} V
\end{gathered}
$$

where $W_{\text {ext }}$ and $W_{\text {elastic }}$ are the energies from the external force and elastic deformation stored in the RVE, respectively. $F_{k}$ is the reaction force at reference node, $d u_{k}$ is the applied differential displacement at that node, $\bar{E}_{i i}=\bar{\sigma}_{i i} / \bar{\varepsilon}_{i i}$ is the axial stiffness in the direction of loading mode, and $V$ is the total volume of RVE. Note that in Equation (5b), $i$ is the index for loading mode and there is no summation on repeated indices. Also, it must be mentioned that the above simplified equation is only valid if the loading occurs on one deformation mode; i.e., when only one of the $\bar{\varepsilon}_{i i}$ components is imposed and the corresponding $\bar{\sigma}_{i i}$ is the only non-zero stress component. In a linear elastic case, Equation (5) results in:

$$
\bar{E}_{i i}=\frac{F_{j} u_{j}}{\bar{\varepsilon}_{i i}^{2} V}
$$

The strain values in the transverse direction can also be calculated after each simulation, as will be discussed in Section 2.4, in order to estimate the values of corresponding Poisson's ratio. Similarly, for the shear modes, considering that the loading was applied on two reference nodes and engineering shear strain $\left(\gamma_{i j}=2 \varepsilon_{i j}\right)$, one can write:

$$
\begin{gathered}
W_{e x t}=2 \int F_{k} d u_{k} \\
W_{\text {elastic }}=\frac{1}{2} \bar{G}_{i j} \bar{\gamma}_{i j}^{2} V=2 \bar{G}_{i j} \bar{\varepsilon}_{i j}^{2} V
\end{gathered}
$$

where $\bar{G}_{i j}=\bar{\sigma}_{i j} / \bar{\gamma}_{i j}$ is the effective shear stiffness and the repeated indices of $i$ and $j$ refer to the loading mode with no summation. Finally, from the conservation of energy in shear mode:

$$
\bar{G}_{i j}=\frac{F_{k} u_{k}}{2 \bar{\varepsilon}_{i j}^{2} V}
$$

Note that Eqs. (6) and (8) will be directly employed in Section 3 to find the elastic moduli of the R-UCAL elements at different fiber volume fractions. 


\subsection{FEA of the whole UD yarn models}

\subsubsection{Geometry and mesh}

Three full-scale yarn models of size matching Figure 1 (1600x500 pixels/0.85x0.26 mm) were created for comparison purposes. For these models, the macro-scale yarn was assumed to contain infinite out-of-plane length (i.e., long-fiber composite) but with a free perimeter (i.e., no traction with the surrounding boundaries). The geometries were meshed using $3 \mathrm{D}$ continuum elements.

(i) Yarn model RI (low fiber volume fraction $\sim 0.26$, random yarn model, Figure 4a): This model was created by randomly selecting R-UCAL elements, tying them together, and meshing using Abaqus. It was assumed that the specimen was sufficiently large to be considered for a large-scale simulation and thus could be simulated as a single consolidated yarn model with random fiber distribution.

(ii) Yarn model R2 (high fiber volume fraction 0.47, random yarn model, Figure 4b): This model was created using the same approach as in (i) with difference being that library elements with higher volume fractions of fiber were used so that the selected cross section had a volume fraction very similar to that of the actual scanned yarn in Figure 1.

(iii) Yarn model S: This comprehensive model (Figure 4c) having a fiber volume fraction of $\sim 0.47$, which was also verified experimentally on the test sample using ASTM D3171 burn-off test, was created from the actual image of the scanned yarn in Figure 1. Specifically, the fiber phase was segmented from the composite, and then both the fiber and matrix were directly meshed using the ScanIP $^{\mathrm{TM}}$ software. The result of meshing directly from the scanned yarn was that, due to limitations in the scanning resolution, some fibers could not be individually identified and instead appeared as (attached) fiber 'bundles' of varying shape and size. Similar challenges have been reported in the literature regarding the micron level spatial resolution of XMT images for composite materials with close [11]. In Section 3, it will be shown how such artifacts translate into variations in effective macro-scale mechanical properties. 


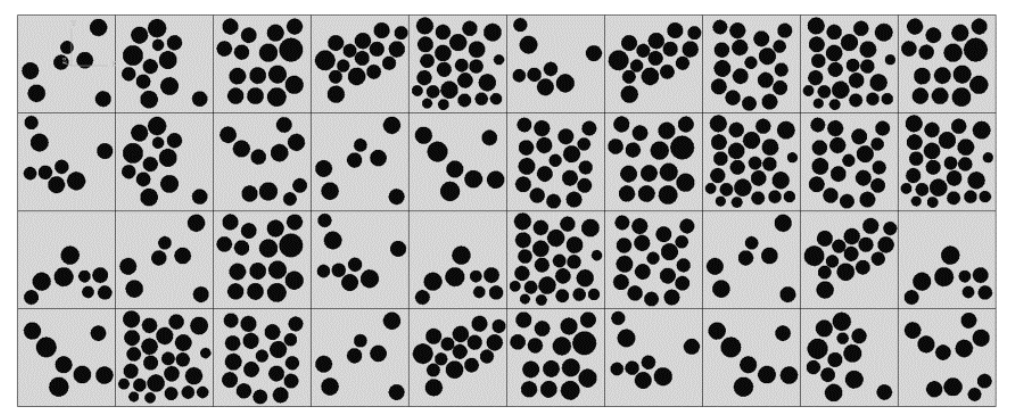

(a)

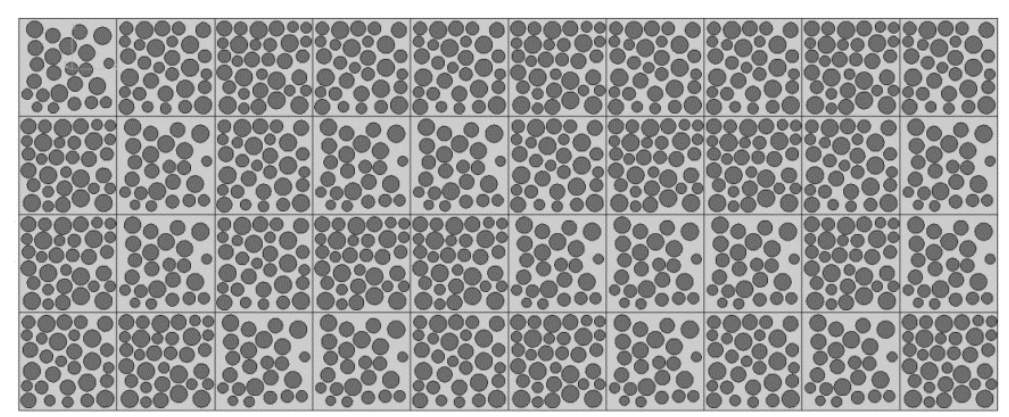

(b)
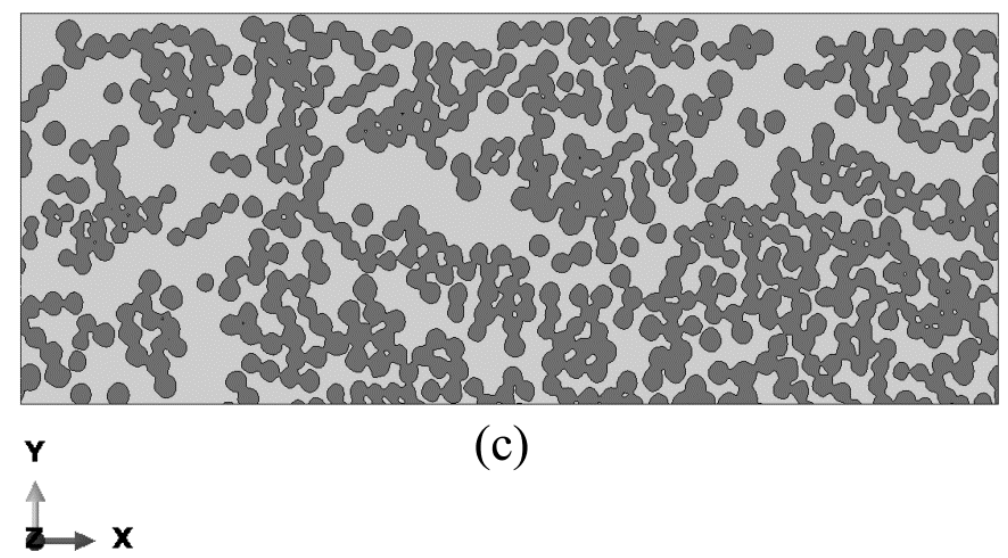

(c)

Figure 4 (a) A full size micro-model of the yarn generated by randomly tying R-UCAL library elements in Figure 2 with a total fiber volume fraction of 0.2612 , (b) full size micro-model of the yearn generated from the library elements but having a fibre volume fraction very similar to that of the actual image $\left(\mathrm{V}_{\mathrm{f}}=0.4765\right)$, and (c) scanned whole-yarn model generated from the $\mathrm{x}$-ray micro-computed tomography image, segmented and meshed in ScanIP ${ }^{\mathrm{TM}}$ and imported to FEA $\left(\mathrm{V}_{\mathrm{f}}\right.$ $=0.4773$ ). These three models in the text are frequently referred to as $R 1, R 2$, and $S$ models, respectively. 


\subsubsection{Simulation procedure and analysis methodology}

The yarn models R1, R2, and S were assumed to simulate an infinitely long yarn that is free on the sides. Therefore, their surfaces were modelled as a free perimeter, and not using the periodic boundary conditions that were imposed on individual R-UCAL elements. As shown in the Appendix, the periodic boundary condition is vital for simulating the deformation of the RVE elements but its effect on the whole-structure (macro-size) model is trivial, especially when the size of the full structure is much larger relative to the RVE.

\section{(i) Normal response and Poisson's ratios}

The axial modulus along the fibers for the three yarn models were extracted by fixing one side of the yarn section and then imposing displacement along the fiber direction on the opposite side. One corner was fixed in order to avoid rigid body motion. For the transverse axial stiffness, one of the side surfaces was constrained and displacement was applied on the opposite side. Moreover, in order to induce a plane strain state and because this geometry represented an infinitely long yarn, the two surfaces normal to the fiber direction, i.e. normal to $\boldsymbol{n}_{3}$ and $-\boldsymbol{n}_{3}$, were constrained in the third direction during transverse loading. Figure 5 shows the boundary conditions used for applying axial/transverse tension on the yarn's section.

The corresponding normal stresses, required for finding the effective elastic properties, were determined via summation of the nodal reaction forces on the loading surface divided by the surface area. This nominal stress for loading along fibers can be used to find the axial stiffness as $\bar{E}_{33}=\bar{\sigma}_{33} / \bar{\varepsilon}_{33}$. However, for transverse loading, the full 3D compliance tensor must be evaluated due to the fact that the section is constrained along the third direction. The general formula for the compliance matrix in an orthotropic media is: 


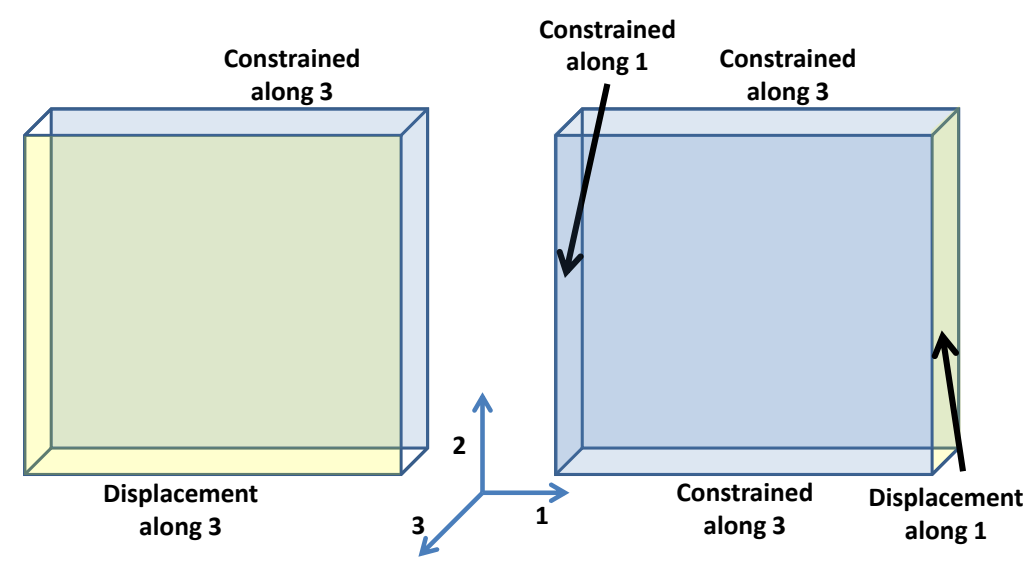

(a)

(b)

Figure 5 Boundary conditions on the yarn element faces for normal loading modes; (a) axial tension along fibers (in 3-direciton), and (b) transverse tension along direction 1.

$$
\left\{\begin{array}{l}
\bar{\varepsilon}_{11} \\
\bar{\varepsilon}_{22} \\
\bar{\varepsilon}_{33} \\
\bar{\gamma}_{12} \\
\bar{\gamma}_{23} \\
\bar{\gamma}_{13}
\end{array}\right\}=\left[\begin{array}{ccccccc}
\bar{S}_{11} & \bar{S}_{12} & \bar{S}_{13} & 0 & 0 & 0 \\
\bar{S}_{21} & \bar{S}_{22} & \bar{S}_{23} & 0 & 0 & 0 \\
\bar{S}_{31} & \bar{S}_{32} & \bar{S}_{33} & 0 & 0 & 0 \\
0 & 0 & 0 & \bar{S}_{44} & 0 & 0 \\
0 & 0 & 0 & 0 & \bar{S}_{55} & 0 \\
0 & 0 & 0 & 0 & 0 & \bar{S}_{66}
\end{array}\right]\left\{\begin{array}{l}
\bar{\sigma}_{11} \\
\bar{\sigma}_{22} \\
\bar{\sigma}_{33} \\
\bar{\tau}_{12} \\
\bar{\tau}_{23} \\
\bar{\tau}_{13}
\end{array}\right\}
$$

Assuming a loading along $i(1$ or 2$)$ direction $\left(\bar{\sigma}_{j j} \neq 0\right)$, while $j(2$ or 1$)$ surfaces are traction-free $\left(\bar{\sigma}_{j j}=0\right)$, the strains are given by:

$$
\begin{gathered}
\bar{\varepsilon}_{i i}=\bar{S}_{i i} \bar{\sigma}_{i i}+\bar{S}_{i 3} \bar{\sigma}_{33} \\
\bar{\varepsilon}_{j j}=\bar{S}_{j i} \bar{\sigma}_{i i}+\bar{S}_{j 3} \bar{\sigma}_{33} \\
\bar{\varepsilon}_{33}=0=\bar{S}_{3 i} \bar{\sigma}_{i i}+\bar{S}_{33} \bar{\sigma}_{33}
\end{gathered}
$$

Finally, an algebraic system of equations can be established [20] and solved to obtain the corresponding transverse stiffness and Poisson's ratio. This requires finding $\bar{\sigma}_{i i}$ (for $\left.i=1,2\right)$ and $\bar{\sigma}_{33}$ (for each of these two cases, respectively, $i=1,2$ ) from the reaction forces on the corresponding surfaces, having $\bar{S}_{33}=1 / \bar{E}_{33}$ from the axial response along the fiber direction, and assuming symmetry in the compliance matrix. 


\section{(ii) Shear response}

The application of a pure shear mode, $\bar{\gamma}_{i j}$, implies that the surface normal to $-\boldsymbol{n}_{i}$ be fixed and that displacement be applied along $u_{j}$ on the opposite surface $\boldsymbol{n}_{i}$, as shown in Figure 6 . Moreover, when shear displacement is applied along the fiber direction $u_{3}$, as in Figure 6, in order to avoid bending in surfaces $\boldsymbol{-} \boldsymbol{n}_{j}$ and $\boldsymbol{n}_{j}$, they require asymmetry boundary condition along the $j$ direction $(j=1$ or 2$)$. On the other hand, when applying the shear loading along $u_{1}$ or $u_{2}$, since the UD yarn is assumed to have a section with infinite length along fibers, $-\boldsymbol{n}_{3}$ and $\boldsymbol{n}_{3}$ surfaces should remain under symmetry along the third direction to ensure the plane strain condition $\left(u_{3}=0\right)$. After running each shear simulation, the nominal shear stress was found through summation of the nodal traction forces on the surface containing the displacement boundary condition. Knowing the imposed level of the applied displacement, the shear strain and subsequently the shear stiffness were calculated. Through additional simulations, where the choice of surface was reversed (i.e. imposing $\bar{\gamma}_{j i}$ instead of $\bar{\gamma}_{i j}$ ), it was found that the numerical results were not noticeably affected for the yarn models $R 1$ and $R 2$ ( $<1 \%$ difference). However, the choice of shearing surface could considerably affect the results of shear tests on the yarn model $S$. This will be further discussed in Section 3.

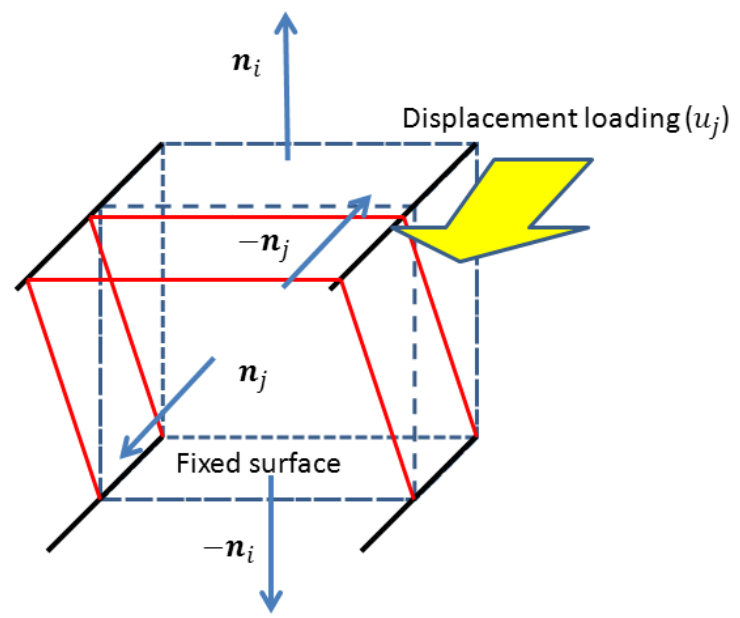

Figure 6 Schematic of applying shear mode, $\gamma_{i j}$ : surfaces normal to $-n_{j}$ and $n_{j}$ are under asymmetry boundary condition along $j$ while $-n_{i}$ section is fixed and loading displacement is applied on $n_{i}$ section along $u_{j}$. 


\subsection{Elastic constants of unidirectional fiber-reinforced composites}

The effective elastic properties determined from the R-UCAL RVE's and the whole UD yarn models can be compared against classical mechanics approaches, as well as the Halpin-Tsai (H-T) equations [21] as follows.

(a) The longitudinal elastic modulus $E_{33}$ given by the rule of mixtures is:

$$
E_{33}=E_{f} V_{f}+E_{m} V_{m}
$$

where $V_{f}$ is the fiber volume fraction, $V_{m}$ is the matrix volume fraction, and $E_{f}$ and $E_{m}$ are the elastic moduli of the individual fiber and matrix materials, respectively.

(b) The major Poisson's ration, $v_{31}$, which is assumed to be identical to $v_{32}$ in a UD material at macro-level (transversely isotropic), is given by:

$$
v_{31}=v_{32}=v_{f} V_{f}+v_{m} V_{m}
$$

(c) Based on a mechanics approach, the transverse elastic modulus $E_{11}$, which is equal to $E_{22}$ for a transversely isotropic media, is given by:

$$
\frac{1}{E_{11}}=\frac{V_{f}}{E_{f}}+\frac{V_{m}}{E_{m}}
$$

However, the H-T equation for the transverse elastic modulus, $E_{11}$ and also $E_{22}$, is:

$$
\frac{E_{11}}{E_{m}}=\frac{E_{22}}{E_{m}} \frac{1+\xi \eta V_{f}}{1-\eta V_{f}}
$$

where $\xi$ is the reinforcing factor and depends on loading conditions as well as the fiber packing geometry, and is merely found/fitted empirically. $\eta$ is given as:

$$
\eta=\frac{\left(\frac{E_{f}}{E_{m}}\right)-1}{\left(\frac{E_{f}}{E_{m}}\right)+\xi}
$$

The numerical value of Eq. (13) lies between the extreme case of $\xi=0$ (in which case it is simplified to the series-like model of Eq. 10), and $\xi=\infty$ (in which case it is simplified to the parallel-like model of Eq. 12) [22]. 
(d) The H-T equation for in-plane shear modulus, $G_{31}$, is given by:

$$
\frac{G_{31}}{G_{m}}=\frac{1+\xi \eta V_{f}}{1-\eta V_{f}}
$$

where $\eta$ is given as:

$$
\eta=\frac{\left(\frac{G_{f}}{G_{m}}\right)-1}{\left(\frac{G_{f}}{G_{m}}\right)+\xi}
$$

(e) The transverse Poisson's ratio, $v_{12}$, may be approximately calculated from (Halpin [23]):

$$
v_{12} \cong 1-\frac{E_{1}}{G_{12}}
$$

(f) The transverse Poisson's ratio, $v_{13}$, can be found by [21]:

$$
\frac{v_{13}}{E_{11}}=\frac{v_{31}}{E_{33}}
$$

It is worth adding that there exist several other analytical homogenization methods [21] including Variational Bounding Technique, Self Consistency Method, and Mori-Tanaka. The Halpin-Tsai equations and classical rule of mixtures have been selected as two example analytical methods, similar to an earlier study [24]. Further, these other methods, unlike the Halpin-Tsai equations, do not account for variation in the geometry of composite particulates. In all cases, the analytical models assume that the fibers never touch one another, which is not practically the case at higher volume fraction of composite [21]. In contrast, there is no limitation on the geometry, size and constituent material properties of a given composite in numerical (FE-based) homogenization methods.

\section{Results and Discussion}

The results consisting of the elastic moduli and Poisson's ratios extracted from the deformation simulations of the R-UCAL elements as well as those from the whole yarn models $R 1, R 2$ and $S$ are shown in Figures 7-9 as a function of fiber volume fraction. In these simulations, the properties of polypropylene and E-glass material (Table 1) have been used. 
Table 1: Material properties of E-glass and Polypropylene used in the micro-level simulations

\begin{tabular}{|l|c|c|c|}
\hline \multicolumn{1}{|c|}{ Property } & Unit & E-glass & Polypropylene \\
\hline Tensile Modulus & $\mathrm{GPa}$ & 73.1 & 1.4 \\
\hline Poisson's ratio & - & 0.22 & 0.3 \\
\hline Axial shear modulus & $\mathrm{GPa}$ & 30.19 & 0.54 \\
\hline
\end{tabular}

Figure 7 shows the axial modulus, $E_{33}$, and the transvers modulus, $E_{11}$, (which is equal to $E_{22}$ for a transversely isotropic UD yarn) of all the R-UCAL elements as well as the random and scanned yarn models. As can be seen in Figure 7a, the axial modulus predicted by modeling of the R-UCAL elements under random fiber distribution follows very closely the classical rule of mixtures for all the library elements, as well as the whole-yarn models $R 1$ and $R 2$ and the actual scanned model $S$. This is in support of the earlier findings on the minimal effect of fiber randomness on axial elastic stiffness of composite fiber tows [28]. In contrast, the calculated values for the transverse modulus are quite different and overestimated as compared to those obtained from the classical mechanics approach as shown in Figure 7b. The R-UCAL and whole-yarn models $R 1$ and $R 2$ instead follow the predictions of the $\mathrm{H}-\mathrm{T}$ equation due to its semi-analytical empirical nature. Interestingly, however, the transverse modulus of the yarn model $S$ is considerably higher than the random media with similar fiber volume fraction. This could be due to the fact that some of the fibers were attached together in this scanned yarn media, causing continuous/perfectly bundled fiber regions that affect the load transfer capacity, or in other words the stiffness under a given deformation level, in transverse direction. This effect is analogous to the 'shear wall' of buildings, which counters the influence of lateral load acting on the structure. From a composite manufacturing perspective, this effect also means that if the fibers are not welldistributed into the matrix but instead are uncontrollably bundled (e.g., during open manual layup), a severe deviation from the theoretical values of composite properties in transverse directions could potentially result. 


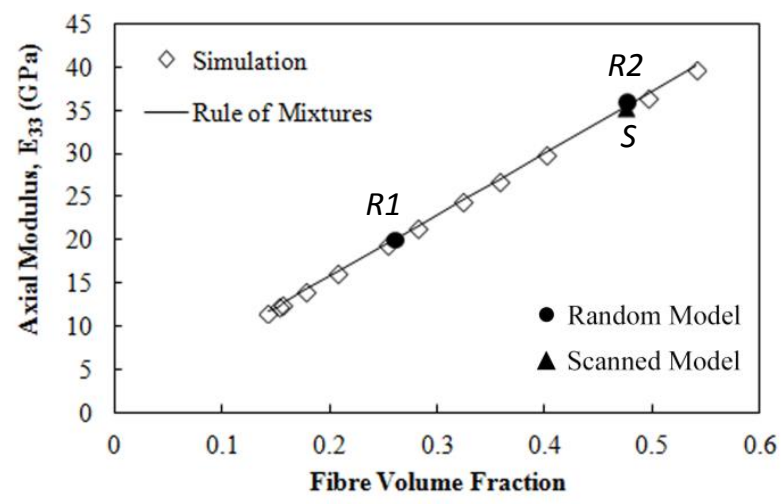

(a)

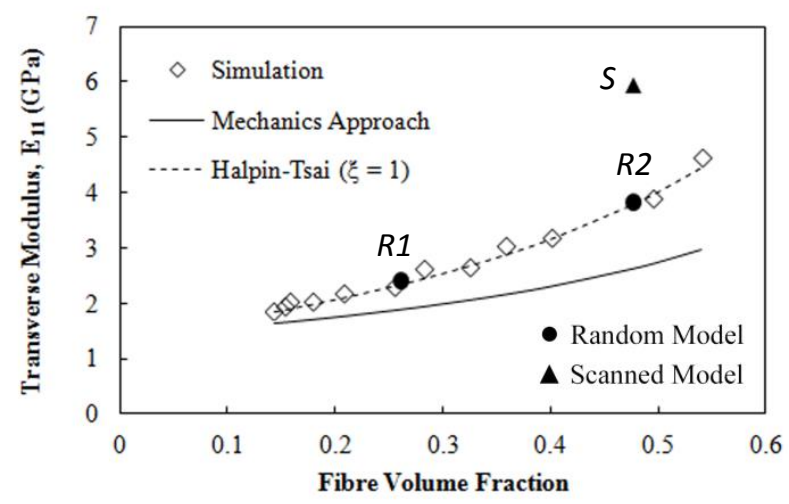

(b)

Figure 7 (a) Axial modulus $\left(E_{33}\right)$, and (b) transvers modulus $\left(E_{11}=E_{22}\right)$ of all the library unit cells as well as the whole-yarn random models $R 1, R 2$, and the actual scanned model $S$. The graph includes data obtained from the simulation as well as the theoretical predictions for comparisons.

The Poisson's ratios resulting from the simulations are presented in Figure 8. Figure 8a shows the transverse-to-axial Poisson's ratio, $v_{31}$ (which is identical to $v_{32}$ in a transversely isotropic UD material) from the simulation models as well as the mechanics approach. Data points from the R-UCAL element library are in good agreement with the mechanics approach, while the whole yarn model $S$ shows a lower $v_{32}$ compared to the other media. The $v_{12}$ prediction is shown in Figure 8b. In general, the calculated values show a scattered distribution over the fiber volume fraction, i.e., no particular trend, and even yarn systems with similar fiber volume fractions but different fiber distribution randomness show different $v_{12}$ Poisson's ratios. Namely, the $v_{12}$ value was 0.39 for the yarn model $R l$, while the library element with similar volume fraction showed a $v_{21}$ value of 0.35 . Hence, clearly the fiber distribution plays a strong role in the Poisson's ratio of composite materials, especially in the transverse-to-transverse component. For this elastic 
constant, the Halpin-Tsai equation also greatly underestimates the simulated findings. Finally, the calculated values of $v_{23}$, shown in Figure $8 \mathrm{c}$, were also in line with the theory for all the library elements and whole yarn models, except for the yarn model $S$. This is presumed to be again a consequence of having fiber bundles (shear wall artifact) instead of individually dispersed fibers in the scanned media from XMT.

Figure 9 shows the in-plane and out-of-plane shear moduli of the library elements and yarn models. As can be seen, the in-plane shear modulus, $G_{13}$, of both the library and whole-yarn random media obeyed the Halpin-Tsai equation with $\xi=1.5$, while the out-of-plane shear modulus, $G_{12}$, also matched the Halpin-Tsai equation with $\xi=0.5$. However, the whole yarn model $S$ again showed a considerable over-estimation in shear moduli.

The results presented in Figures 7-9 on the micro-scale modeling of UD fiberglass/polymer yarns via two approaches (R-UCAL elements and the whole yarn models) confirmed that fiber distribution, including both their radii and location relative to each other, could affect considerably the effective properties of composites. It was shown that when the fibers are individually separated, the properties of the entire composite yarn can be simulated by using small unit cells while imposing a random distribution of fibers, e.g., from an actual XMT image of the yarn. The computational advantage here is that the unit cell approach with appropriate periodic boundary conditions gives almost the same response of the whole yarn as long as the selected volume fraction of the representative RVE (unit cell) and the actual yarn are the same. This is shown throughout Figures 7-9, as the whole yarn models $R 1$ and $R 2$ are aligned with the predictions of the R-UCAL elements. However, when the fibers are bundled such as in the whole yarn model $S$, the predicted elastic constants and Poisson's ratio are quite different. In this case, the presence of fiber bundles significantly affects the stress transfer among the fibers, as well as stress transfer between the fibers and the matrix, and consequently influences the composite effective properties in the transverse direction. The effect of such fiber entanglement on the stress transfer/distribution within the yarn is further investigated in Figure 10, in which a comparison of the von-Mises stress between the whole yarn model $S$ and the yarn model $R 2$ of similar fiber volume fraction $(\sim 0.47)$ is provided. As can be seen, the stress contours show significantly higher values in the fiber entangled regions. This indicates greater stress transfer. To more closely visualize fiber entanglement, a high magnification (1500X) image of the same test sample, obtained using a Stereo Optical Microscope, is shown in Figure 11. 
Finally, one can conclude from Figures (7) to (9) that the modified macro-level rules of mixture via the Halpin-Tsai equations are fairly consistent in predicting the average values of effective UD yarn properties over different volume fractions, even in the presence of fiber distribution randomness at micro-level. However, the results obtained in this work indicate that the main limitation of this theoretical model is that a different reinforcing factor $\xi$ should be estimated for each type of material constant/loading mode. This is practically impossible without testing and modeling of the actual yarn. The exception to this limitation, however, is the prediction of the yarn's axial modulus $\left(E_{33}\right)$ and the axial Poisson's ratio $\left(v_{31}\right)$, which do not require $\xi$ and are equivalent to the classical rule of mixtures. 


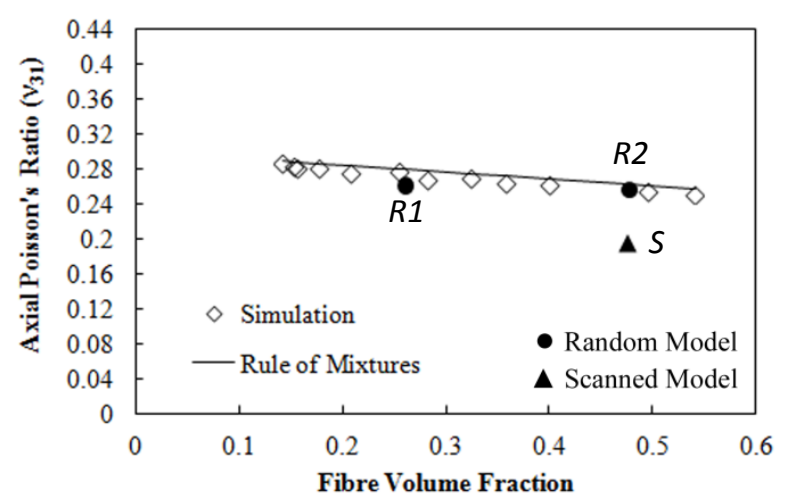

(a)

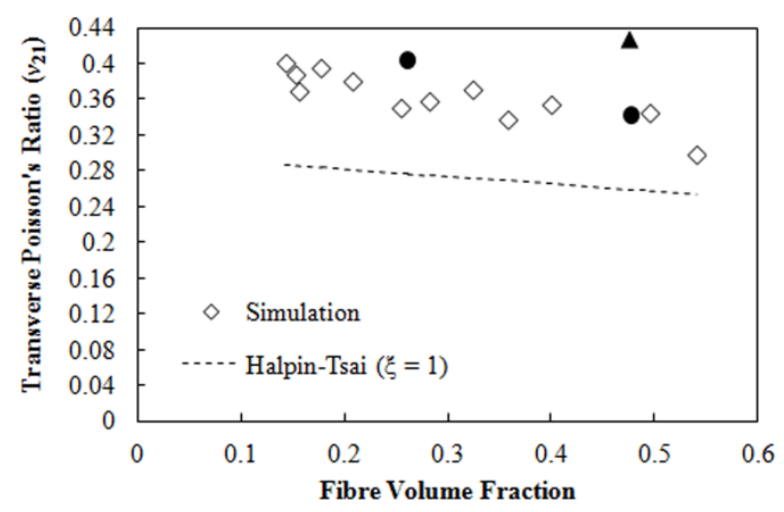

(b)

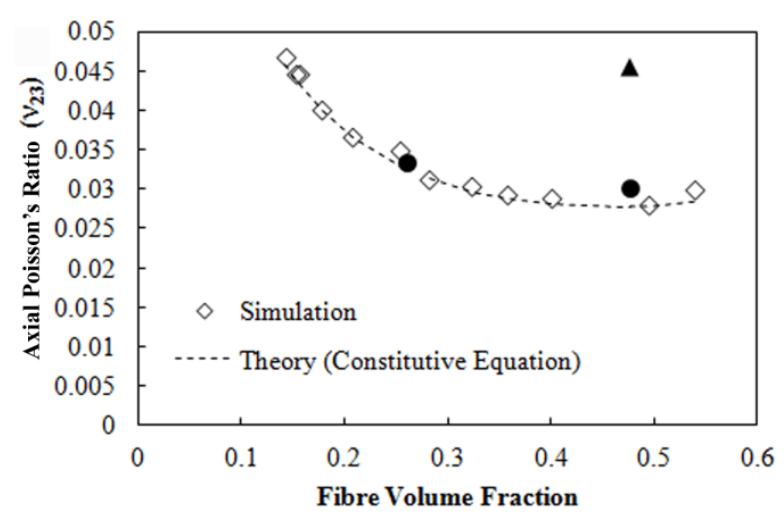

(c)

Figure 8 (a) Axial Poisson's ratio $\left(v_{31}=v_{32}\right)$, (b) the transverse Poisson's ratio $\left(v_{21}\right)$, and (c) the axial Poisson's ratios $\left(v_{23}=v_{13}\right)$ of the R-UCAL library elements, whole-yarn random models $R I$ and $R 2$ and the scanned model $S$. Theoretical values have also been shown for comparisons. 


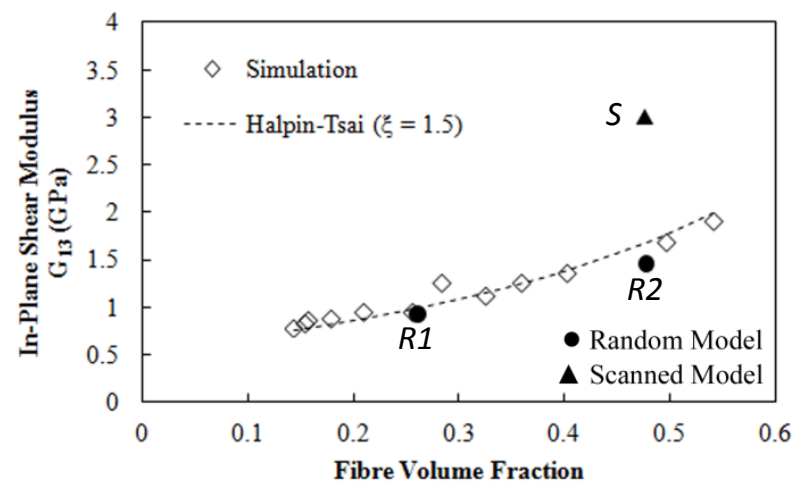

(a)

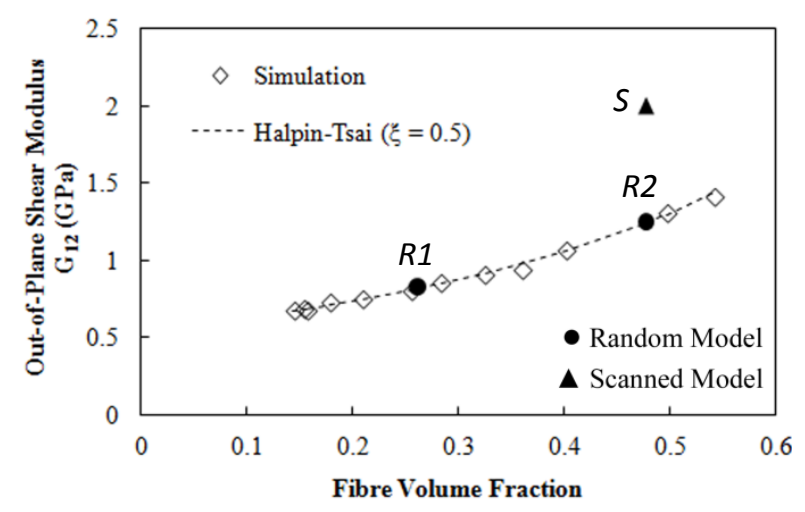

(b)

Figure 9 (a) The in-plane $\left(G_{13}\right)$ and (b) out-of-plane $\left(G_{12}\right)$ shear moduli of the library units, random models (yarn models $R 1$ and $R 2$ ) and scanned model (yarn model $S$ ). Theoretical values have also been plotted for comparisons. 


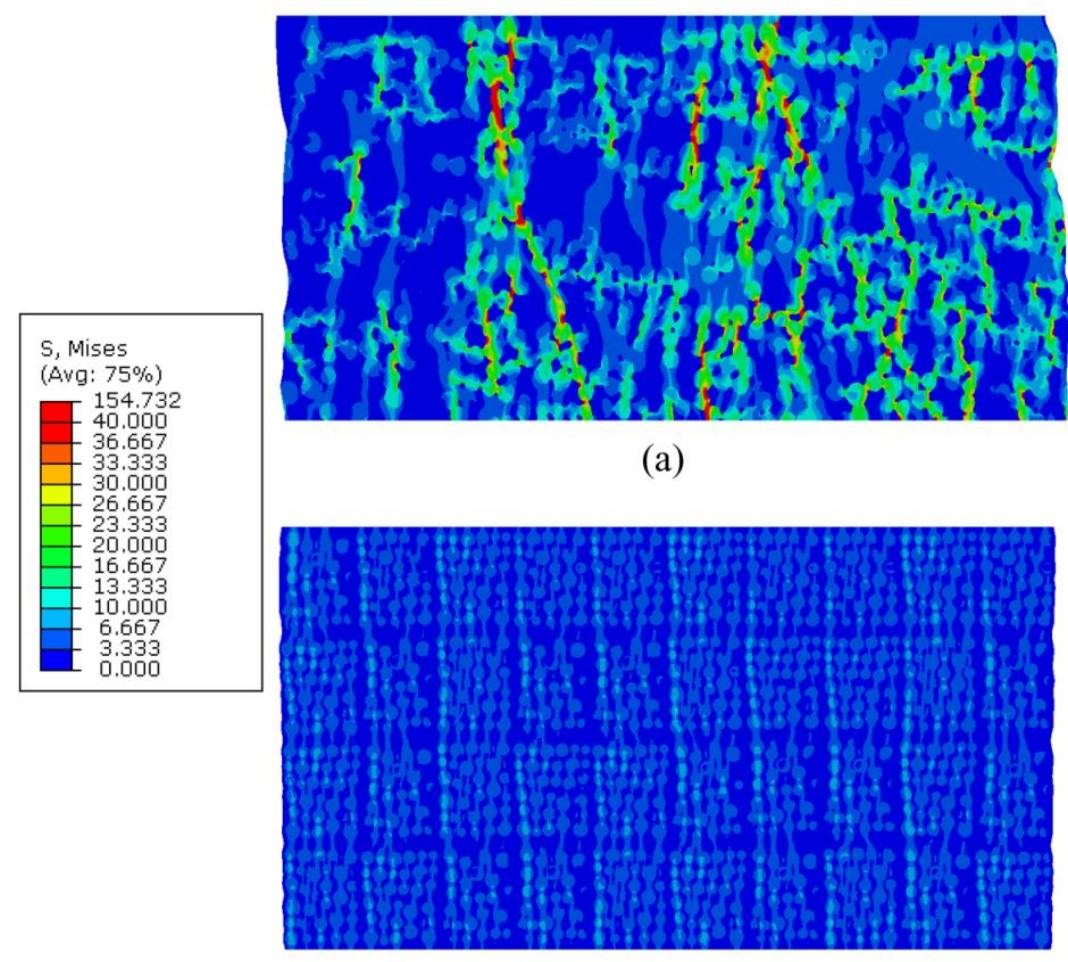

(b)

Figure 10 Von Mises stress contours of the (a) scanned whole-yarn model $S$, and (b) random whole-yarn model $R 2$ with a similar fibre volume fraction ( 0.47). A significantly greater stress values in the fibres-entangled regions can be seen in the stress contours of the XMT scanned model, indicating a higher/overestimated stress transfer capacity within the material microstructure.

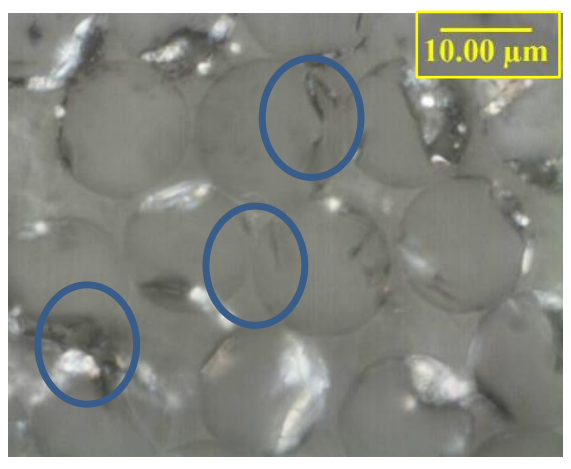

Figure 11 High magnification (1500X) image of the yarn sample with a Stereo Optical Microscope; notice the fuzzy boundaries between the fibers inside example blue marked regions, suggesting very careful post-processing of scanned images during RVE modeling in order to avoid artificial shear-wall effects in the subsequent FE analysis as shown in Figure 10. 


\section{Concluding remarks}

The micro-scale modeling of a UD fiberglass/polymer yarn was performed under random fiber distribution. It was shown that the properties of the composite yarn are influenced by fiber distribution including their radii and location relative to each other. Overall, the results revealed that random fiber distribution did not significantly alter the axial and transverse elastic moduli as well as the transverse-to-axial Poisson's ratio and shear moduli (i.e., the micro-simulations followed the macro-level theories). However, fiber distribution strongly affected the transverseto-transverse Poisson's ratio since yarns of different fiber distributions, under a similar volume fraction, resulted in very different values that did not match the theory. The deviation between RUCAL models and the full scale yarn model could also be related to the effect of RVE sizes [30, 31, 32]. Regardless, when using an image-based or XMT scanned yarn model, the status of fibers was found to have a significant effect on the simulated mechanical properties at the micro-scale. For example, when an image of the yarn was used for direct modeling, i.e. yarn model $S$, the fibers could not be separated completely during image processing and thus consisted of complex shapes. This geometry change in the reinforcement significantly affected the stress transfer amongst the fibers and between the fibers and the matrix, resulting in an overestimation of the transverse mechanical properties at macro-scale.

The results of this study have shown that, while the classical rule of mixture can be used for predicting the axial modulus and transverse-to-axial Poisson's ratio of a UD yarn with random fiber distribution, this theory cannot be used for transverse moduli and other Poisson's ratios that show a non-linear variation with fiber volume fraction. For the latter properties, the Halpin-Tsai equations work fairly well, though they are limited since the reinforcing factor $\xi$ varies as a function of loading mode. However, if a specific composite processing creates conditions where fibers become actually merged or bonded together forming a shear-wall in the cross-section of the yarn, then a direct image-based modeling at the micro-scale is needed to confidently simulate the material's response under different loading modes.

In the future, it may be worthwhile to create a library of randomized unit cells for other fiber/matrix yarn types for similar micro-level FE simulations in order to make the yarn's effective mechanical properties readily available for subsequent macro-level simulations under different 
fiber volume fractions. The inclusion of fiber path randomness (misalignment/ waviness) through XMT and full-scale yarn modeling with porosity effects would also be of high interest for further accounting for micro-level uncertainties and composite defects. Finally, while the presented work might be considered as a first step towards studying the effect of fiber clustering evident in micrographs, this may greatly affect the macroscopic response of composites when going beyond the elasticity limits. There is not much information available on the latter subject yet and it could be recommended as another critical future work.

\section{Acknowledgements}

Funding for this research was provided by the Natural Sciences and Engineering Research Council (NSERC) of Canada, the Composites Research Network (CRN), and the Canada Foundation for Innovation (CFI). Highly valuable comments and feedbacks from the anonymous reviewers are acknowledged. The authors would also like to acknowledge the work of Dr. M. Alemi Ardakani from CRN, who provided the Stereo Optical microscope image of fiber entanglement.

\section{References}

[1] Mair R. I., Fiber reinforced polymers-From Aerospace to Infrastructure. ATSE (Australian Academy of Technological Sciences and engineering) Focus, 1999, No. 107, 5-6/99.

[2] Boisse P., Finite element Analysis of Composite Forming, In: Long, A.C. (ed.), Composite Forming Technologies, Woodhead Publishing, Cambridge, 2007, 46-79.

[3] Dobrich O., Gereke T. and Cherif C., Modelling of textile composite reinforcements on the micro-scale, AUTEX Researhc Journal, 2014; 14(1):28-33.

[4] Xia Z., Zhou C., Yong Q. and Wang X., On selection of repeated unit cell model and application of unified periodic boundary conditions in micro-mechanical analysis of composites, International Journal of Solids and Structures, 2006; 43:266-278.

[5] Aboudi J., Mechanics of composite materials: A unified micromechanical approach, Elsevier Science Publishers, Amsterdam, 1991.

[6] Nemat-Nasser S. and Hori M., Micromechanics: Overall properties of heterogeneous materials, Elsevier Science Publishers, Amsterdam, 1993.

[7] Wolodko J. D., Xia Z., Ellyin F., Analysis of $\mathrm{Al} / \mathrm{Al}_{2} \mathrm{O}_{3}$ metal matrix composites under biaxial cyclic loading using a digital image based finite element method, Materials Science and Technology, 2000; 16:837-842. 
[8] Laurin F., Carrere N. and Maire J. F., A multiscale progressive failure approach for composite laminates based on thermodynamical viscoelastic and damage models, Composites Part A: Applied Science and Manufacturing, 2007; 38(1):198-209.

[9] Laurin F., Carrere N., Huchette C. and Maire J. F., A multiscale hybrid approach for damage and final failure predictions of composite structures, Journal of Composite Materials, 2013; 47(2021):2713-2747.

[10] Blacklock M., Bale H., Begley M. and Cox B., Generating virtual textile composite specimens using statistical data from micro-computed tomography: 1D tow representations for the binary model, Journal of the Mechanics and Physics of Solids, 2012; 60:451-470.

[11] Alghamdi A., Mummery P. and Sheikh M. A., Multi-scale 3D image-based modelling of a carbon/carbon composite, Modelling Simul. Mater. Sci. Eng., 2013; 21:085014 (13pp).

[12] Badel P., Vidal-Salle E., Maire E. and Boisse P., Simulation and tomography analysis of textile composite reinforcement deformation at the mesoscopic scale, Composites Science and Technology 2008; 68:2433-2440.

[13] Bakhvalov N. and Panasenko G., Homogenisation: Averaging processes in periodic media, Mathematics and its applications, Mathematical problems in the mechanics of composite materials, Kluwer Academic Publishers, The Netherlands, 1989.

[14] Takano N., Uetsuji Y., Kashiwagi Y. and Zako M., Hierarchical modeling of textile composite materials and structures by the homogenization method, Model Simul Mater Sci Eng, 1999; 7:207231.

[15] Peng X. and Cao J., A dual homogenization and finite element approach for material characterization of textile composites, Composites: Part B, 2002; 33:45-56.

[16] Xia Z., Zhang Y. and Ellyin F., A unified periodical boundary condition for representative volume elements of composites and applications, International Journal of Solids and Structures, 2003; 40:1907-1921.

[17] Badel P., Vidal-Salle E., Boisse P., Computational determination of in-plane shear mechanical behaviour of textile composite reinforcements, Computational Materials Science, 2007; 40: 439448.

[18] Li S., Zhou C., Yu H. and Li L., Formulation of a unit cell of a reduced size for plain weave textile composites, Computational Materials Science, 2011: 50(5):1770-1780.

[19] Sejnoha M. and Zeman J., Micromechanical modeling of imperfect textile composites, International Journal of Engineering Science, 2008; 46(6):513-526.

[20] Mase G. T. and Mase G. E., Continuum Mechanics of Engineers, $2^{\text {nd }}$ edn., New York: CRC Press, 1999.

[21] Kaw A. K., Mechanics of Composite Materials. New York: CRC Press, 1997. 
[22] Jones I. A. and Pickett A. K., Mechanical properties of textile composites, In: Design and manufacture of textile composites, Edited by Long A. C., Woodhead Publishing, Cambridge, 2005.

[23] Halpin J. C., Primier on Composite Materials Analysis, $2^{\text {nd }}$ edn., Technomic, Lancaster, PA, 1992.

[24] Węglewski W., Bochenek K., Basista M., Schubert Th., Jehring U., Litniewski J., and Mackiewicz S., Comparative assessment of Young's modulus measurements of metal-ceramic composites using mechanical and non-destructive tests and micro-CT based computational modeling, Computational Materials Science, 2013; 77:19-30.

[25] Pan Y., Iorga L., and Pelegri A., Numerical generation of a random chopped fiber composite RVE and its elastic properties, Composites Science and Technology, 2008; 68(13): 2792-2798.

[26] Abdin Y., Lomov S.V., Jain A., Van Lenthe G.H., Verpoest I., Geometrical characterization and micro-structural modeling of short steel fiber composites, Composites Part A, 2014; 67: 171180.

[27] Nov'ak J., Ku`cerov'a A., and Zeman J., Compressing random microstructures via stochastic wang tilings, Physical Review E, 2012; 86:040104.

[28] Zeman J., and `Sejnoha M., Numerical evaluation of effective elastic properties of graphite fiber tow impregnated by polymer matrix, Journal of the Mechanics and Physics of Solids, 2001; 49(1): 69-90.

[29] Michel J. C., Moulinec H., and Suquet P., Effective properties of composite materials with periodic microstructure: A computational approach, Computer Methods in Applied Mechanics and Engineering, 1999; 172: 109-143.

[30] Trias D., Costa J., Turon A., and Hurtado J.E., Determination of the critical size of a statistical representative volume element (SRVE) for carbon reinforced polymers, Acta Materialia, 2006; 54(13): 3471-3484.

[31] Stroeven M., Askes H., and Sluys L.J., Numerical determination of representative volumes for granular materials, Computer Methods in Applied Mechanics and Engineering, 2004; 193 (3032): 3221-3238.

[32] Zeman J., and Sejnoha M., From random microstructures to representative volume elements, Modelling and Simulation in Materials Science and Engineering, 15(4), 2007; S325-S335.

[33] Pahr D. H., and Zusset P. K., Influence of boundary conditions on computed apparent elastic properties of cancellous bone, Biomechanics and Modeling in Mechanobiology ,2008; 7(6):463476. 


\section{Appendix: How important is to apply periodic boundary condition on RVE and/or whole yarn models}

In order to evaluate the effect of imposing a periodic boundary condition on the RVE as well as on the whole-structure modeling approaches (which were both used during UD yarn's micro-FEA in the current work), a rudimentary case study was conducted in this appendix as a side example. First, an arbitrary 2D plane-strain RVE was constructed. Then this RVE was modeled under four different scenarios: (1) using the periodic boundary conditions all around; (2) no periodicity; (3) a larger structure made of the repetition of the latter RVE ( 5 by 5) with a periodic boundary condition; and (4) a similar large structures but without periodicity. The contours of the transverse stress distribution and deformed shape of the 2D model for each case is depicted in Figure A1. Moreover, the ensuing (homogenized) transverse Young's modulus values are shown in Figure A2. As it can be seen, the periodic and non-periodic RVE models are very different $(\sim 35 \%)$; however, the whole structure model responses with or without periodicity are fairly similar. Therefore, the periodic boundary condition is deemed to be the necessary feature of microlevel RVE as it can provide a good representation of the large (macro) structure with or without a periodic boundary. Detailed studies on the effect of boundary condition in RVE analysis of other materials types, including orthotropic or nearly orthotropic such as bone, can be found in [33]. 


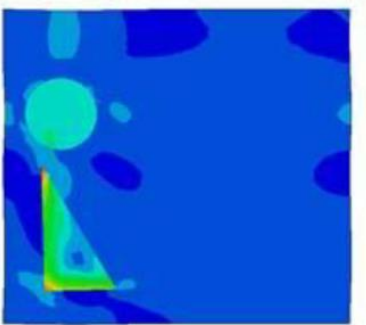

(a)

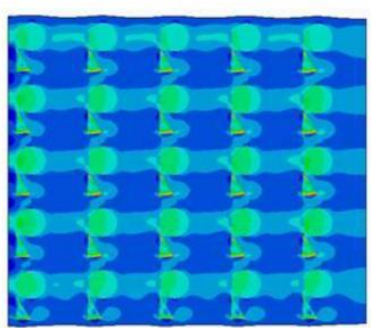

(c)

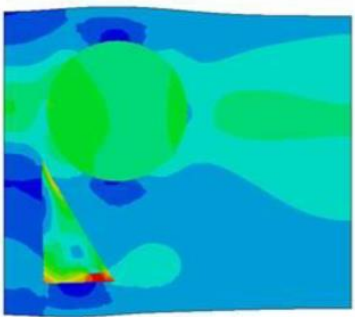

(b)

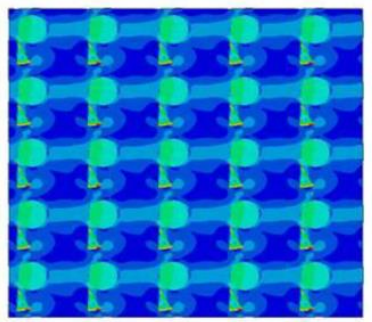

(d)

Figure A1 Deformed shape of (a) an example unit cell with periodic boundary condition, (b) the same unit cell without periodic boundary condition, (c) a larger (full size) structure with $5 \times 5$ size of the initial unit cell and without periodic boundary condition, and (d) the same large structure but with periodic boundary condition. All the models are under uniaxial tension in the horizontal direction. Notice that the boundary lines of model (b) are not straight after deformation.

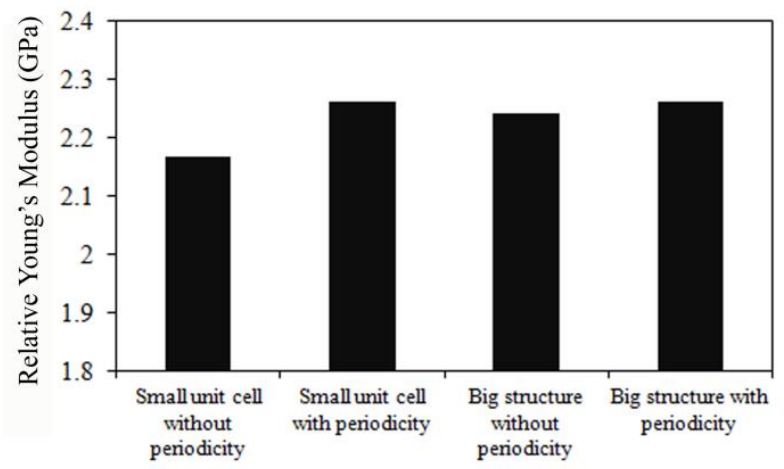

Figure A2 Relative transverse Young's moduli of the models shown in Figure A1. 